\title{
Modelo matemático de calidad de agua aplicado en la microcuenca del río Tabacay
}

\section{Water quality mathematical model applied in the watershed of the river Tabacay}

\author{
Carlos Matovelle \\ Universidad Católica de Cuenca \\ Cuenca, 010150, Ecuador \\ cmmatovelleb@ucacue.edu.ec
}

\begin{abstract}
Resumen
El agua es uno de los recursos naturales más apreciados en la actualidad, mantener la calidad es hoy en día uno de los objetivos principales de las administraciones públicas que lo controlan. Para esto se han creado herramientas como los modelos de calidad de agua que permitan una adecuada gestión del recurso. Se ha desarrollado un modelo de calidad de agua para la microcuenca del río Tabacay por el elevado valor social e institucional que tiene al ser la única fuente de abastecimiento para la ciudad de Azogues y estar sometida a una gran contaminación por las actividades de ganadería, agricultura y falta de sistemas de saneamiento. Para el desarrollo del modelo se utiliza el software libre de la Agencia de Protección Ambiental estadounidense denominado Water Quality Analysis Simulation Program (WASP). Para llegar al desarrollo final del modelo se analiza y valora la información disponible tanto de calidad como de caudales y geometría de la zona, se establecen los parámetros y constantes a ocupar en las simulaciones de acuerdo al sistema que se analiza, en los primeros resultados se observa variaciones de los datos simulados con los medidos por lo que se plantea técnicas para la depuración de los modelos de calidad de agua aplicados en las zonas andinas ecuatorianas para disminuir el grado de error que pueden tener en las simulaciones mediante la aplicación de nuevos estudios para la determinación de parámetros adecuados y metodologías que permitan obtener modelos aplicables a estas zonas.
\end{abstract}

Palabras clave: Agua, Modelos de calidad, Tabacay, Sistemas andinos.

\begin{abstract}
Water is nowadays one of most valued natural resources; maintaining its quality is currently one of the main objectives of the public administrations that exert control over it. For this purpose, some tools such as water quality models have been created in order to allow a proper management of this resource. A water quality model has been developed for the Tabacay micro river basin because of its high social and institutional value, as it is the only water provision source for the city of Azogues and is subjected to high pollution levels caused by livestock, agriculture and lack of sanitation systems. To develop the model, the free software of the US Environmental Protection Agency called Water Quality Analysis Simulation Program (WASP) is used. In order to achieve the final model development, the available information of water quality, as well as water flow and geometry of the area are analyzed and evaluated; the parameters and constants to be used in the simulations are established depending on the system being analyzed, in the first results, variations of the simulated values with respect to the measured ones were observed, which is why the use of techniques for the depuration of water quality models applied to Ecuadorian Andean areas are suggested, with the intent of reducing the error degree in the simulations, by means of the application of new studies for determining the appropriate parameters and methodologies that allow to attain models which can be applicable to these areas.
\end{abstract}

Key words: Water, Quality models, Tabacay, Andean Systems.

\section{INTRODUCCIÓN}

$\mathrm{E}$ L agua en la actualidad se ha constituido en un bien muy preciado debido a la escasez con la que se encuentra en buen estado y a que muchas de las actividades económicas están ligadas a este recurso.

El estado de un río se puede determinar con respecto al grado de alteración que presenta de acuerdo a sus condiciones naturales [1].
Se debe tener en cuenta que la calidad del agua depende del uso para el cual va a ser destinada y está ligada a varios parámetros físicos, químicos y biológicos con límites de concentraciones establecidos. Entonces un agua puede tener una buena calidad para un fin pero esa misma agua no puede ser apta para otro uso.

La contaminación del agua siempre ha estado ligada a las actividades del hombre ya que esta se ha utilizado 
para el vertido de sus residuos por su gran capacidad de purificación pero, el desarrollo ha hecho que exista un aumento demográfico y por lo tanto un aumento en la demanda y la presencia de sustancias contaminantes más difíciles de autodepurar.

\section{A. Contaminación en ríos sudamericanos}

La diferencia de la problemática entre los países desarrollados y los países en vías de desarrollo es muy evidente, los problemas de contaminación en América Latina tienen un origen de tipo social, económico, cultural y legislativo. Mientras los países desarrollados se enfocan en realizar planes para la vigilancia, protección y recuperación de sus recursos hídricos, los países en vías de desarrollo se encuentran solventando trabas legales para poder operar en ámbitos como el control de vertidos en cuerpos de agua.

EL $70 \%$ de la población de América Latina no tienen acceso a sistemas hidrosanitarios que permitan el tratamiento de aguas residuales y estas vuelven a los cuerpos de agua sin haber sido depuradas, el $80 \%$ de la población vive en las ciudades y una gran parte en asentamientos cercanos a fuentes contaminadas convirtiéndose esto en un problema medio ambiental y de salud pública [2].

\section{Modelos DE CALIDAd DE AGUA COMO HERRAMIENTA EN LA TOMA DE DECISIONES}

La calidad del agua depende de varios factores naturales aunque el factor principal que influye en la disminución de la calidad son las actividades humanas . Es necesario buscar herramientas que permitan mejorar la gestión de la calidad del recurso hídrico, dentro de la búsqueda de estas herramientas aparece la modelación matemática.

Un modelo es un conjunto de conceptos y ecuaciones que permiten acercarse a la realidad de un fenómeno, aunque los modelos son conceptos con fundamentación teórica la necesidad de herramientas computacionales ha permitido la creación de formulaciones predefinidas sobre diferentes casos específicos [3].

La necesidad de disponer medidas de control que permitan cumplir los límites de vertido a los cuerpos de agua y el comportamiento de la contaminanción ha obligado ha revisar muchos de los criterios que se utilzan tradicionalmente. Parte importante de esta estrategia es la utilización de modelos que caractericen la dispersión y transporte de los contaminantes en cauces naturales [4].

El enfoque clásico para la gestión de la calidad del agua es, una vez desarrollado y calibrado, utilizar el modelo para predecir la concentración de los contaminantes para distintas alternativas de tratamiento.

\section{A. Balances de materia para la modelación de calidad}

El núcleo de cualquier modelo de calidad de agua son los balances de materia. Los balances se aplican a cada una de las sustancias objeto de interés. Pueden ser contaminantes químicos, oxígeno disuelto, microorganismos, etc., según el

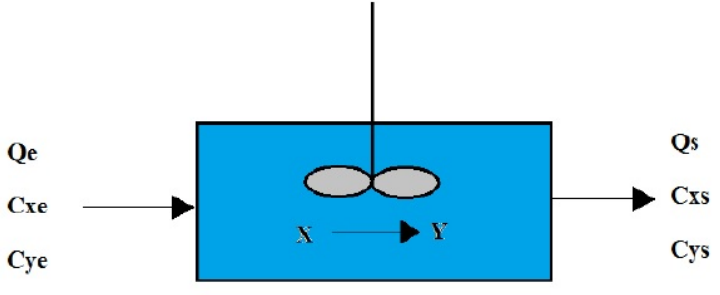

FIG. 1. Esquema de sistema completamente mezclado.

tipo de problema en estudio. Dichas sustancias constituyen los "componentes del modelo" [5].

En sistemas completamente mezclados ha de cumplirse el balance de masas que no es más que un conteo de las entradas, salidas, reacciones y acumulación de materia en un volumen de control. Fig. 1

De ahí ha de cumplirse la ecuación fundamental de conservación de la materia:

Acumulación $=$ Entradas - Salidas \pm Generación

La Ecuación (1), representa la variación de concentración de un contaminante que se encuentra en un elemento diferencial de volumen al cabo de un determinado tiempo.

$$
\begin{array}{r}
\frac{\partial C}{\partial t}=-\frac{\partial}{\partial x}\left(u_{x} C\right)-\frac{\partial}{\partial y}\left(u_{y} C\right)-\frac{\partial}{\partial z}\left(u_{z} C\right)+ \\
\frac{\partial}{\partial x}\left(E_{x} \frac{\partial C}{\partial x}\right)+\frac{\partial}{\partial y}\left(E_{y} \frac{\partial C}{\partial y}\right)+\frac{\partial}{\partial z}\left(E_{z} \frac{\partial C}{\partial z}\right)+ \\
S_{k}
\end{array}
$$

donde $C$ representa la concentración, $t$ el tiempo, $u_{x}, u_{y}, u_{z}$ son los componentes del vector velocidad. $E_{x}, E_{y}, E_{z}$ son los componentes del tensor de dispersión y $S_{K}$ representa la fuente o sumidero del contaminante.

\section{B. Simplificaciones para sistemas naturales}

La mejor manera de representar un sistema es ocupando las tres dimensiones, pero resulta muy complejo y costoso obtener la información necesaria para alimentar este tipo de modelo además de la alta capacidad computacional que esto requeriría; se debe decidir si todo este esfuerzo para obtener un modelo tridimensional compensa los resultados que se pretende obtener desde el punto de vista de calidad del agua.

De aquí que se puede realizar simplificaciones a los sistemas naturales sin variar considerablemente los resultados que se obtiene al final. Para ríos podemos trabajar con un sistema unidimensional o bidimensional dependiendo de las características del mismo.

La mayoría de veces se considera a los ríos sistemas unidimensionales porque su longitud es muchas veces mayor a su anchura, de esta consideración podemos obtener una simplificación presente en la Ecuación (2)

$$
\frac{\partial C}{\partial t}=-\frac{\partial}{\partial x}\left(u_{x} C\right)+\frac{\partial}{\partial x}\left(E_{x} \frac{\partial C}{\partial x}\right)+S_{k} .
$$


Otra consideración que se puede realizar en los ríos es que no existe transporte dispersivo por lo que se elimina el término de este tipo de trasporte, resultando la Ecuación (3)

$$
\frac{\partial C}{\partial t}=-\frac{\partial}{\partial x}\left(u_{x} C\right)+S_{k}
$$

Con las simplificaciones realizadas es posible modelar cualquier tipo de contaminante presente en un río. Este modelo simula los contaminantes no conservativos tradicionales, es decir, aquellos que sufren una transformación en el medio en el que se encuentran. Resulta de vital importancia conocer cada uno de los procesos de aparición/desaparición que sufren los contaminantes con sus determinadas expresiones de modelación para poder acercarse a la realidad de los procesos ocurrentes.

\section{Metodología Del tRABAJO}

En este apartado se analiza y califica la información proporcionada por la Empresa de Agua Potable y Alcantarillado (EMAPAL) para determinar los datos y series temporales a utilizar para realizar las simulaciones. Se analizan los datos de calidad que van a ser empleados en las simulaciones y solamente de las quebradas que tienen captaciones de agua para la planta de potabilización.

A continuación se explica el proceso realizado para la modelación de la calidad del agua en la microcuenca del Tabacay. La Figura 2 recoge una síntesis de los trabajos que componen esta metodología.

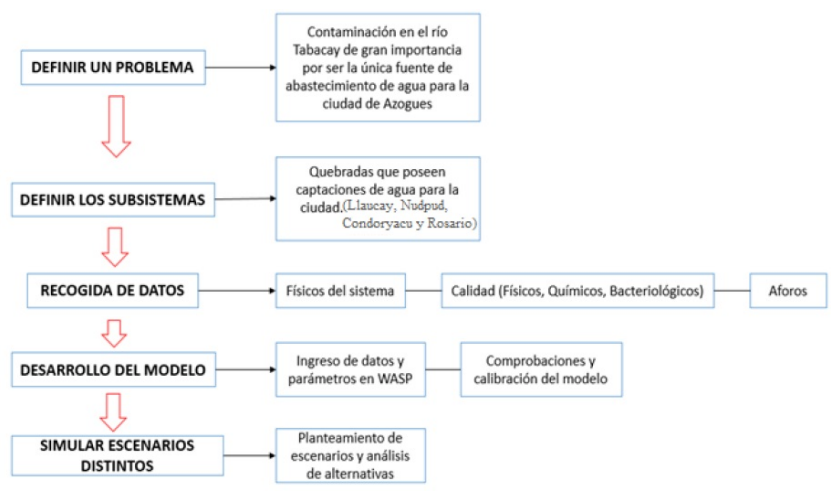

FIG. 2. Metodología propuesta para el desarrollo del trabajo.

La definición del problema y la elección de los subsistemas se realizan teniendo en cuenta aspectos importantes como:

- Garantizar la calidad del recurso agua para abastecimiento urbano.

- Garantizar el recurso agua para la agricultura y ganadería.

- Valor ecológico.

- Datos disponibles.

La metodología plantea una identificación de la zona de interés, la microcuenca del río Tabacay y las quebradas que la forman tienen una claro acción antrópica por lo que justifica la necesidad de una herramienta de gestión adecuada. La prioridad es garantizar el recurso hídrico para la población, ya que es la única fuente de agua por lo que se elige realizar un modelo para las cuatro quebradas que son utilizadas para captación de agua, esto reduce la zona de estudio. Además de eso ya que se intenta garantizar el recurso para la población los tramos de río a ser modelados serán desde su naciente hasta el embalse de captación de agua.

Luego de haber establecido la problemática y los subsistemas que se analizan, el trabajo consiste en la recopilación de datos disponibles que sean necesarios para el desarrollo del modelo.

- Con los datos de calidad se puede realizar el modelo con los parámetros de interés, se analiza sólidos suspendidos, materia orgánica, oxígeno disuelto, nitrógeno y coliformes.

- Con los aforos se analiza el régimen de caudales, necesarios para el desarrollo del modelo. La variación temporal de los caudales depende de régimen hidrológico además de eso la actividad humana también contribuye a la variación del caudal, en este caso por la canalización para consumo de poblaciones y agricultura.

Los datos que se posee de la red de calidad y caudales son en las captaciones; series mensuales consecutivas desde el mes de octubre del año 2009 hasta el año 2014. Se realiza una valoración y depuración de los datos disponibles eliminando los valores en blanco que van a ser usados para la calibración del modelo.

En la Figura 3 se presentan los puntos de las captaciones y por lo tanto de la red de calidad existente.

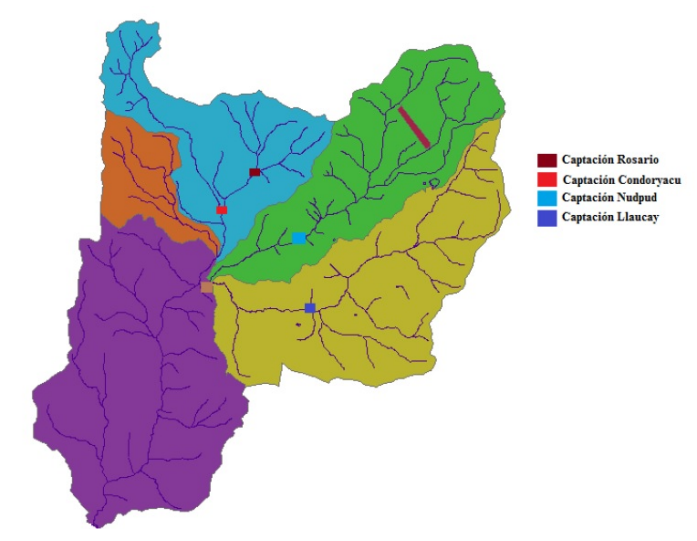

FIG. 3. Estaciones de monitoreo de calidad en el río Tabacay.

\section{A. Descripción del Water Quality Analysis Modeling Sys- tem (WASP)}

El modelo WASP desarrollado por la Agencia de Protección Ambiental (EPA) de los Estados Unidos nos permite analizar y predecir las respuestas de calidad de agua a los 
fenómenos naturales y a la contaminación artificial que sufren los cuerpos de agua.

El WASP es un modelo que permite simular el comportamiento dinámico de un sistema acuático, incluyendo la columna de agua y los bentos. Este modelo permite analizar sistemas en 1, 2 y 3 dimensiones y para un variado número de contaminantes [6].

Los tres componentes del modelo son: WASP para el transporte de masas; EUTRO de oxígeno disuelto, nutrientes y cinéticas de algas; y TOXI para sustancias tóxicas. WASP es capaz de realizar un análisis en tiempo variable o estado estacionario y en una, dos o tres dimensiones. Además las nuevas versiones poseen un módulo para análisis de mercurio y de calor.

\section{DESARRollo DEL MODELO}

Para el desarrollo del modelo en el rio Tabacay se analiza un sistema de una dimensión segmentando cada una de las quebradas que son analizadas. Los parámetros que intervienen en las simulaciones son analizados y justificados dentro de los rangos típicos y el correcto funcionamiento del modelo. Para obtener una modelación en la cual el sistema se comporte de la manera más cercana a la realidad se corren simulaciones para las cuatro quebradas que son usadas para captación de agua dentro la microcuenca del río Tabacay, con los datos observados se realizan ajustes de los parámetros que intervienen. Luego de analizar los datos de monitoreos de calidad de agua es evidente destacar que la quebrada Llaucay es la que presenta el mayor grado de contaminación por lo que este artículo se presentan los resultados de este tramo.

\section{A. Segmentación}

La segmentación del río permite dividir por zonas de interés e incluir la geometría de cada una de estas zonas y las relaciones de velocidad de cada segmento. Los datos de mediciones de geometría existentes de la quebrada Llaucay permiten dividirla en 4 segmentos desde aguas arriba, considerando el tanque de captación el último segmento del sistema. No se posee una caracterización de los afluentes que llegan a la quebrada por esto se incluye un segmento adicional en el cual se agrupan estos, considerando que el tramo de interés y de ajuste es el segmento previo a la captación. En la Figura 4 se presenta el esquema de segmentación que se adopta en WASP.

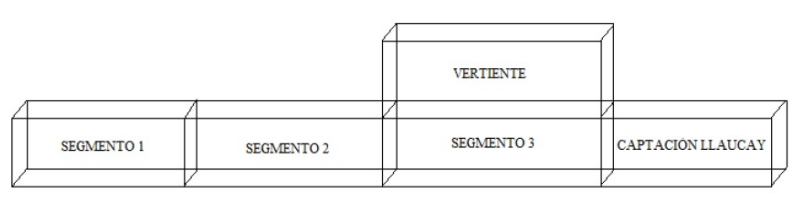

FIG. 4. Esquema de segmentación de la quebrada Llaucay.
Si se analiza un río proveniente de montaña lo normal es encontrar sistemas transitorios, variados y turbulentos pero se realizan suposiciones de regímenes estacionarios para lograr establecer las condiciones iniciales del modelo y luego analizar un sistema que se asemeje más a la realidad.

Entonces bien, al momento de segmentar el río consideramos tramos que tienen dimensiones y pendiente constantes, si una tasa de flujo constante $Q$ entra en el extremo aguas arriba para un período suficientemente largo de tiempo, el agua llegará a una condición de flujo uniforme. La profundidad del tramo y la velocidad del agua también serán constantes y estarán relacionadas al flujo por la ecuación de continuidad [7].

En la Ecuación (4) se puede observar la relación que existe entre caudal, velocidad y la sección transversal del tramo a analizar.

$$
Q=U \cdot A_{c},
$$

donde $Q$ es el caudal del flujo, $U$ es la velocidad media y $A_{c}$ es la sección transversal del río.

Ya que el caudal depende de la velocidad y de la profundidad se necesita de mayor información para determinar los valores de las magnitudes. Para obtener esta información usamos los coeficientes de descarga, cuyas ecuaciones relacionan la velocidad media, profundidad y ancho con el flujo [9], estas ecuaciones son:

$$
\begin{aligned}
U & =a Q^{b}, \\
H & =\alpha Q^{\beta}, \\
B & =c Q^{f},
\end{aligned}
$$

donde $H$ es la profundidad del río, $a, b, \alpha, \beta, c$ y $f$ son constantes empíricas que se determinan a partir de curvas de altura-caudal. Debido a que estos parámetros están interrelacionados, los coeficientes no son totalmente independientes.

La Tabla A. muestra los valores y rangos para los exponentes [7]. Estos valores se utilizan en las simulaciones de caudales que realiza el modelo y son verificados antes de realizar las simulaciones de los contaminantes.

TABLA I

VALORES MEDIOS Y RANGOS DE CORRELACIONES HIDROGEOMÉTRICAS

\begin{tabular}{|c|c|c|c|}
\hline Correlación & Exponente & Valor & Rango \\
\hline Velocidad-Flujo & $b$ & 0.45 & $0.3-0.7$ \\
\hline Profundidad-Flujo & $\beta$ & 0.4 & $0.1-0.6$ \\
\hline Ancho-Flujo & $f$ & 0.15 & $0.05-0.25$ \\
\hline
\end{tabular}

\section{B. Determinación de constantes en WASP}

Un modelo debe ser capaz de reproducir y predecir las variables deseadas con un mínimo número de parámetros y tiempo de cálculo, existen procesos químicos físicos y 
biológicos que están definidos por relaciones estequiométricas y cinéticas para los cuales debemos determinar un cierto número de constantes para que el modelo funcione de manera adecuada.

Para la determinación de constantes en el modelo WASP se dispone de un menú en el cual podemos elegir las opciones con las cuales trabajar y los rangos de valores disponibles a condiciones de temperatura estándar $\left(20^{\circ} \mathrm{C}\right)$.

\section{Degradación de materia orgánica}

La formulación matemática de la de DBO carbonosa fue hecha por Streeter y Phelps con base en la ley empírica de Theriault en la que se indica que la tasa de oxidación bioquímica de materia orgánica es directamente proporcional a la cantidad de materia orgánica biodegradable presente, obedece a una ecuación de primer orden [8].

WASP nos permite elegir constantes de degradación a $20^{\circ} \mathrm{C}$ y de semisaturación ya preestablecidas, esto permite correr las primeras simulaciones para obtener datos de como se comporta el sistema. Con los valores tomados observamos que luego de ejecutar el modelo la constante de degradación para la materia orgánica se encuentra alrededor de $0.3 d^{-1}$. Figura 5 .

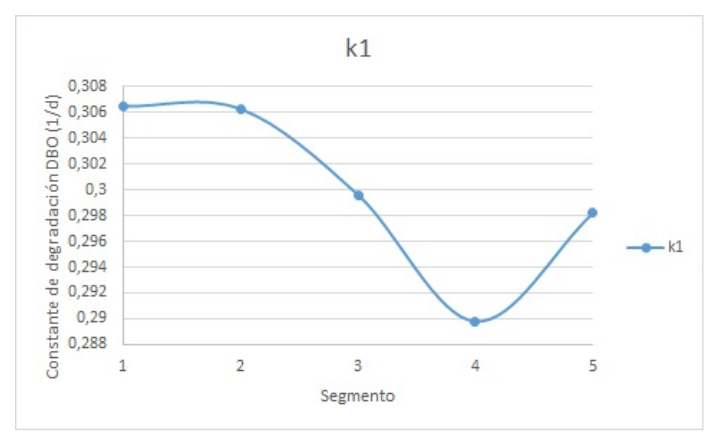

FIG. 5. Valores para la constante de degradación de materia orgánica.

Un rango para la constante de degradación de materia orgánica es 0.05-0.4 $d^{-1}$ [10]; por lo que se asume que el rango en el cual está trabajando la simulación es correcto y los procesos en los cuales interviene materia orgánica en la quebrada de análisis son reales.

\section{Oxígeno disuelto}

En ríos con características andinas la reaireación tiene un papel muy importante en la recuperación del sistema acuático, para determinar la constante de reaireación en la quebrada Llaucay se utiliza el método Covar, además se tiene en cuenta la altura de la microcuenca y la constante de corrección por temperatura.

Para la determinación de la constante de reaireación se utiliza la Figura 6, la cual condensa el método Covar de acuerdo a las características de velocidad y calado de los ríos, la quebrada Llaucay está dentro de las características de la zona Owens-Gibbs.

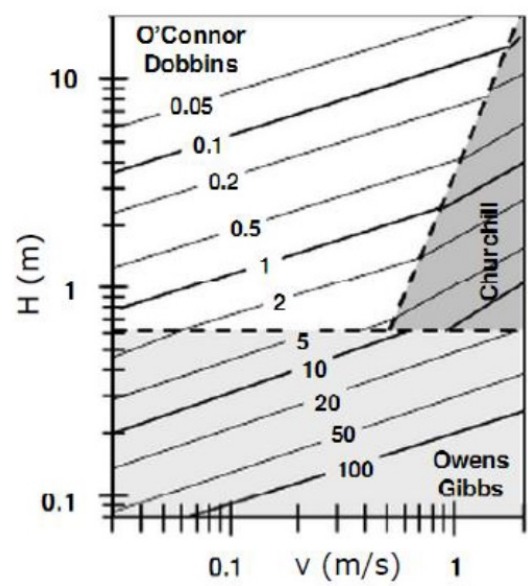

FIG. 6. Determinación de la constante de reaireación por el método Covar para ríos.

Luego de correr las simulaciones con los datos establecidos se obtienen los resultados para las velocidades y la constante de reaireación. Estos resultados se muestran en la Figura 7 y Figura 8 , se observa que los valores de estas magnitudes corresponden con las relaciones que se presentan en la Figura 6. Además se puede observar que en los segmentos de aumento de velocidad también se tiene un aumento en la reaireación del agua. La simulación arroja datos de oxígeno de saturación cercano a los 10 $\mathrm{mg} / \mathrm{l}$ pero en las microcuencas andinas por la altura a la que se encuentren los flujos de agua y particularmente en las quebradas que forman la microcuenca del Tabacay el oxígeno de saturación está alrededor de los $8 \mathrm{mg} / \mathrm{l}$.

Esa consideración se debe tener en cuenta en el momento del ingreso elevado de materia orgánica en el modelo para ajustar la disminución del oxígeno disuelto.

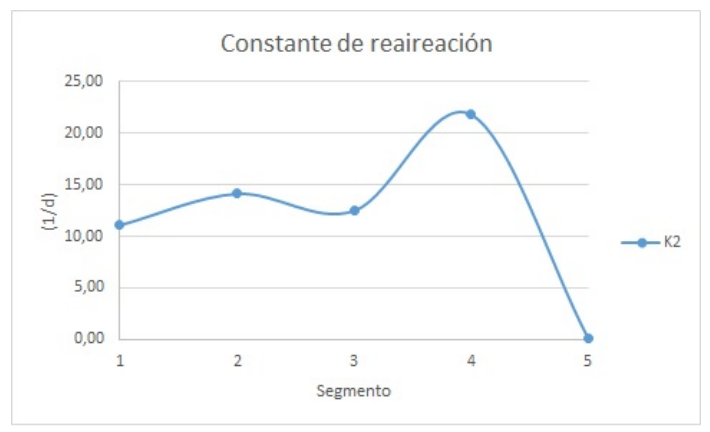

FIG. 7. Valores para la constante de reaireación.

\section{Nitrógeno}

El modelo para calidad del río Tabacay que se presenta contempla las especies de nitrógeno ya que al existir el proceso de nitrificación del amonio se consume oxígeno de la columna de agua provocando problemas de calidad. 


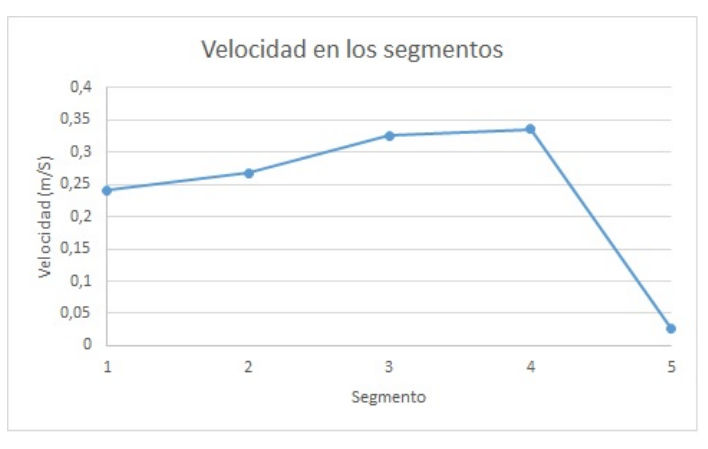

FIG. 8. Valores de velocidad del agua en los segmentos.

Se simula la mineralización del nitrógeno orgánico en amonio, la nitrificación y la desnitrificación; para estos tres procesos marcamos en los menús de WASP las constantes de velocidad de los procesos, los coeficientes de corrección para la temperatura y los coeficientes de semisaturación para cada proceso, las constantes para las cinéticas de todos estos procesos se toman desde el catálogo de WASP.

\section{Cargas externas}

En los puntos anteriores se modela el río sin recibir aportes de contaminantes externos, solo se considera las fronteras y se supone para esta simulación que le agua que ingresa por las fronteras tiene la calidad del estado natural por lo que no recibe aportes de contaminantes, esto permite saber como se comporta el sistema en estado natural.

Se tienen los datos de calidad en la captación que es el último tramo de la segmentación en el modelo y como se observa en puntos anteriores no posee la calidad que se supone tiene el agua que ingresa por las fronteras ya que la red de monitoreo existente solo contempla monitoreo en el tramo previo a la captación. Existe disminución de oxígeno por lo que es necesario la presencia de procesos que consuman este oxígeno, esto supone un ingreso de contaminantes.

Lo que se hace en este punto es suponer las cargas de contaminantes que deben existir para poder llegar a los datos de calidad que posee la captación. A continuación se enumera las suposiciones que realizamos para poder realizar la simulación, esto permite realizar un modelo solamente con la información disponible y son consideraciones que no se alejan de la realidad de los sistemas analizados.

1) El agua que ingresa por las fronteras tiene la calidad del agua en estado natural.

2) Todas las vertientes se agrupan en un solo segmento que llega como aporte en el segmento 3 del modelo.

3) El flujo del río es del $70 \%$ del caudal que llega a la captación y la vertiente es del $30 \%$ del caudal total que llega a la captación.

4) Los contaminantes ingresan por el flujo de la vertiente.

5) La relación entre la concentración de nitrógeno kjenldahl total (NKT) y de materia orgánica que ingresan en la vertiente es 1:10.
6) Los segmentos 1 y 2 se encuentran libres de contaminación.

\section{Resultados}

El objetivo del modelo es reproducir las condiciones que se tienen observadas en la captación suponiendo las condiciones aguas arriba hasta lograr tener los datos observados y simulados con el mínimo error. Luego de realizar varias simulaciones de ajuste para ver el comportamiento del sistema y la carga de contaminantes se obtiene los resultados para la simulación con caudales variables, temperatura variable, materia orgánica, oxígeno disuelto, nitrógeno, sólidos suspendidos, demanda de oxígeno por el sedimento y coliformes fecales, el objetivo de esta simulación es obtener un modelo completo.

El modelo intenta ajustarse a los datos de oxígeno disuelto en las captaciones suponiendo lo que sucede aguas arriba y las cargas que ingresan con la vertiente. El tiempo de simulación es de 26 meses, desde octubre de 2009 hasta noviembre de 2011 ya que en este periodo es donde se posee la mayor disponibilidad de datos.

\section{A. Resultados para oxígeno disuelto y materia orgánica}

Como se puede observar en la Figura 9 se consigue un ajuste que sigue la tendencia de los datos que disponemos, tiene un error absoluto promedio de $1.8 \%$. Al ser el objetivo del modelo ajustarse a las concentraciones de oxígeno en la captación mientras menor sea el error van a ser más certeras las condiciones supuestas aguas arriba.

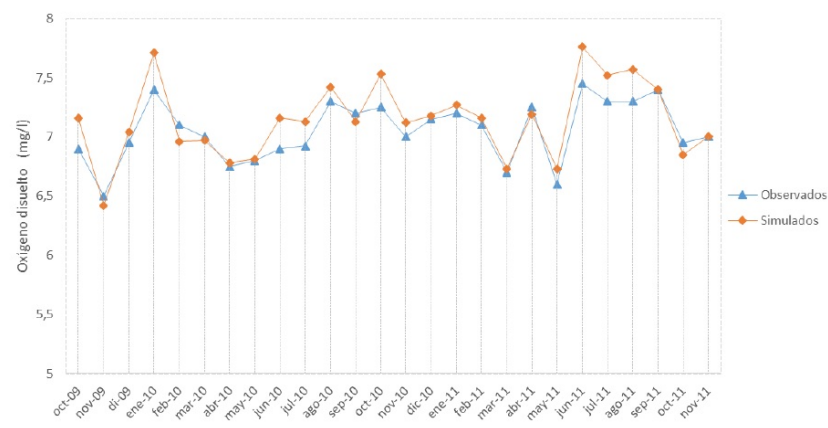

FIG. 9. Comparación de valores de oxígeno disuelto observados y simulados.

Para lograr el ajuste en el oxígeno disuelto en el segmento que representa la captación se debe disminuir la concentración desde el valor de saturación que se dispone para la zona de estudio hasta los datos medidos, para esto en la vertiente ingresan cargas de materia orgánica y de amonio cuya presencia provoca la disminución del oxígeno, además de ingresar con la vertiente nitrógeno orgánico, nitratos, sólidos suspendidos y coliformes fecales.

En la Figura 10 se presentan los resultados para la concentración de materia orgánica simulada y observada en la captación. 


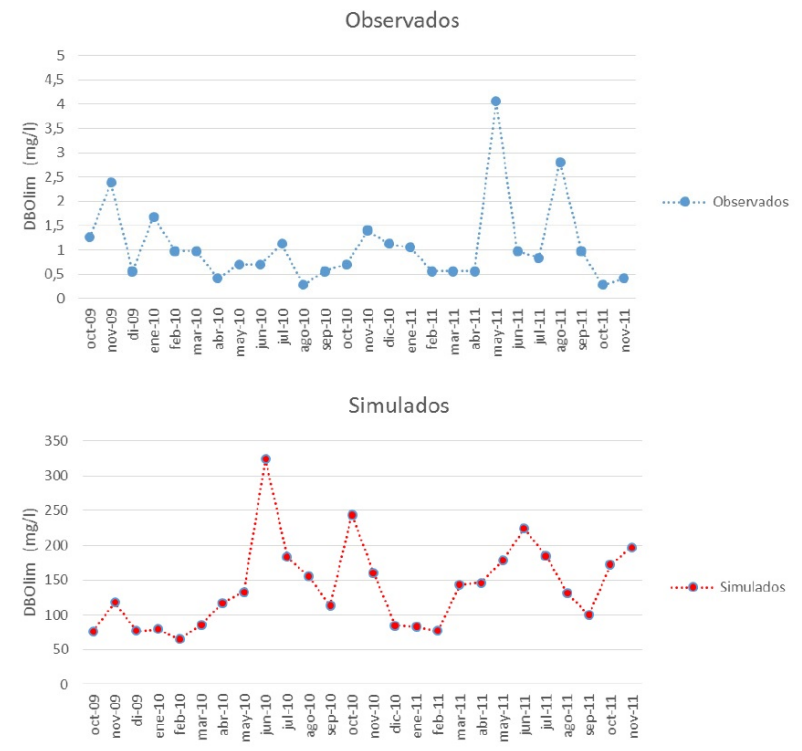

FIG. 10. Concentraciones de materia orgánica medida como DBO límite en la captación.Datos observados vs simulados.

En las dos figuras es evidente la gran diferencia que existe entre las concentraciones simuladas y las observadas. Los valores medidos son típicos para sistemas en los cuales no exista contaminación y para que haya una disminución de la concentración de oxígeno desde el de saturación debe haber presencia de materia orgánica que lo consuma en su degradación.

Los resultados simulados indican que debe ingresar una carga de contaminante elevada que disminuya la concentración de oxígeno disuelto de saturación que simula el modelo, la problemática se presenta porque el modelo da valores de oxígeno de saturación elevados en comparación con los reales, se debe tener en cuenta que estos ríos tienen una gran dinámica y recuperación por lo que hay que considerar que esa disminución es muy poco probable ingresando valores de contaminación bajos.

El oxígeno de saturación de la zona está claramente afectado por la altura por lo que el simulado se aleja de la realidad sin poder corregir ese parámetro en el modelo, se debe colocar valores reales y de esta manera ajustar con menos grado de error el modelo.

\section{B. Resultados para nitrógeno}

El análisis de los resultados de amonio y nitrógeno orgánico en las simulaciones no se puede ajustar a los datos reales que se tienen de las mediciones en las captaciones ya que fueron manipulados para ajustar la concentración de oxígeno disuelto simulado.

Al existir disminución de oxígeno disuelto y ser una microcuenca que recibe vertidos de agua residual urbana y escorrentías de suelos con usos ganaderos y agrícolas existe una probabilidad elevada de especies de nitrógeno, por este motivo la presencia de concentraciones más altas que los datos observados es correcta, aunque para lograr la disminución del oxígeno observado las concentraciones de nitrógeno son elevadas. En la Figura 11 se observa la concentración de amonio tanto en datos simulados como observados. La concentración de amonio que se tienen luego de simular es muy superior a la de los datos.

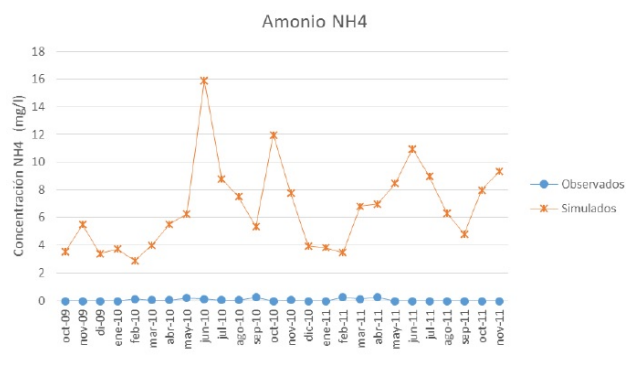

FIG. 11. Comparación de concentraciones de amonio simuladas y observadas en la captación.

Al existir una disminución de oxígeno disuelto en el agua es muy probable la presencia de concentraciones de amonio elevadas que consuman el oxígeno en la nitrificación.

\section{Resultado para sólidos suspendidos}

Una de las mayores problemáticas en la microcuenca del río Tabacay es la carga de sólidos que lleva consigo sus efluentes ya que estos llegan hasta la central de generación eléctrica causando problemas en el turbinaje. Al ser un parámetro que se puede simular independientemente de la concentración de oxígeno que tiene el agua, se puede realizar un ajuste del modelo para que se asemeje lo más posible a los datos observados.

Este ajuste se presenta en la Figura 12, existen algunos meses con concentraciones muy elevadas por lo que se ha divido la gráfica en dos partes, la una con la escala normal y la otra con una escala más pequeña para poder observar el ajuste para las concentraciones.

Como se observa en la figura anterior los datos simulados siguen la tendencia de concentración de sólidos suspendidos. Para que exista esa concentración en la captación hay una carga que ingresa al sistema.

\section{Resultado para coliformes fecales}

La concentración de bacterias dependerá de la carga del vertido y de la tasa de desaparición que posean. Existen varios factores que afectan la velocidad de desaparición de las bacterias fecales, como el efecto de la luz que hace que la velocidad de crecimiento aumente, la adsorción en los sólidos suspendidos que representa un sumidero, la sedimentación que disminuye la concentración en la columna de agua aunque representa un aumento en el sedimento, la temperatura, el $\mathrm{pH}$, depredadores, entre otros factores.

Como la mayoría de las expresiones utilizadas para la modelación de la calidad del agua para la desaparición de las bacterias fecales se utiliza una cinética de primer orden. 

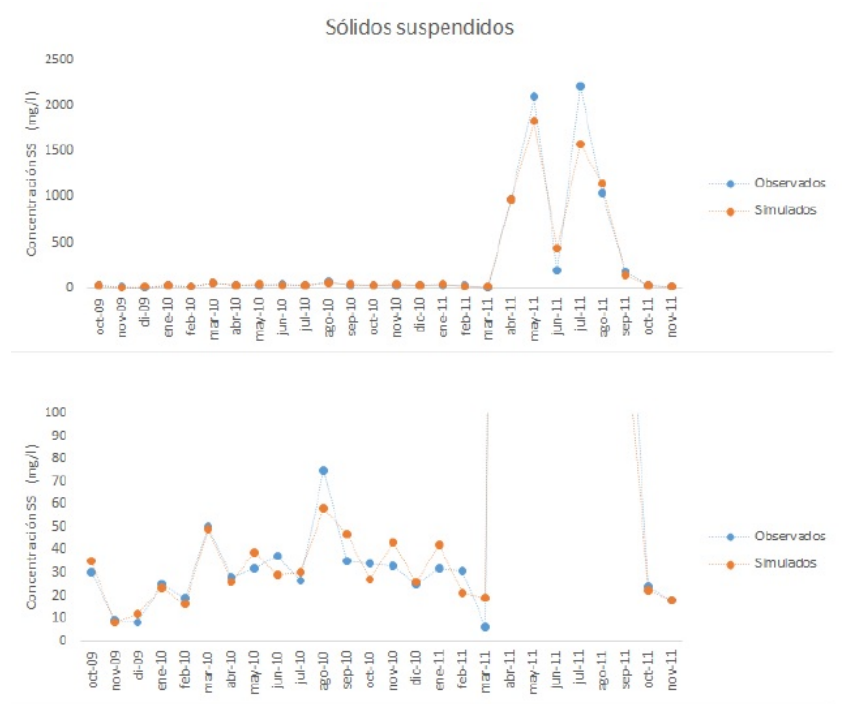

FIG. 12. Comparación de valores de sólidos suspendidos observados y simulados.

$$
\frac{\partial C}{\partial t}=-k C
$$

donde $C$ es la concentración de coliformes y $k$ es la constante de desaparición.

Los valores de $k$ varían mucho a lo largo del día, por lo que resulta complejo la determinación en la simulación. La manera más adecuada para la determinación de esta constante es mediante ensayos de laboratorio o experiencias in situ.

Para el modelo en WASP se utiliza el módulo de simulación para la degradación de la materia orgánica que al igual que la desaparición de bacterias fecales utiliza una cinética de primer orden y se ajusta a los datos observados. La variación de la constante de desaparición simulada en WASP se observa en la siguiente Figura 13

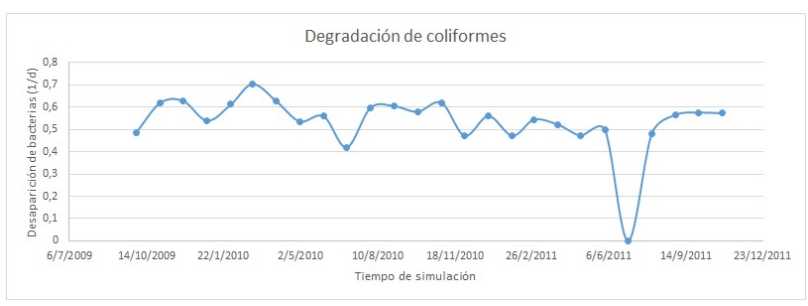

FIG. 13. Constante de desaparición de las bacterias fecales. Simulación

Como se puede observar en la figura anterior la constante maneja rangos medianamente constantes con excepciones puntuales que son los meses en los que aumente notablemente la concentración de coliformes por lo que la tasa de desaparición es prácticamente nula.

Como se puede observar en la figura anterior la constante maneja rangos constantes con una excepción puntual en el mes de junio de 2011 en los que aumente notablemente la concentración de coliformes por lo que la tasa de desaparición es prácticamente nula. Esta evento puede deberse a un vertido puntual de algún contaminante que llevaba una carga bacteriana elevada.

En la siguiente figura se encuentran las concentraciones de bacterias fecales observadas y simuladas, se logra un ajuste correcto en la simulación con error absoluto promedio del $6 \%$.
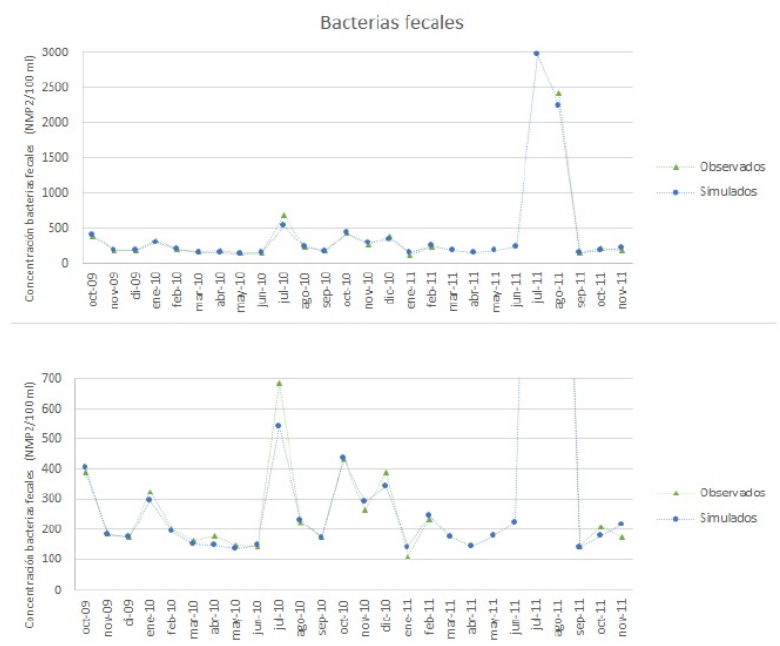

FIG. 14. Comparación de valores de bacterias fecales observados y simulados.

\section{CONCLUSIONES}

Un modelo matemático de calidad de agua debe simular el sistema de manera que todos los componentes que intervengan tengan relación y se ajusten de la mejor manera a la realidad, en el modelo de la microcuenca del río Tabacay luego de ajustar el parámetro de oxígeno disuelto, la concentración de materia orgánica simulada es elevada en comparación con los datos observados, esto indica un error el cual se debe analizar su origen.

Las constantes de las cinéticas para los procesos de modelación fueron obtenidas mediantes simulación, se encuentran dentro de los rangos de trabajo, para el desarrollo de modelos en sistemas que tengan variaciones tan visibles tanto hidrodinámicas como de calidad es necesario caracterizar experimentalmente las constantes cinéticas y disminuir el grado de error posible.

El origen de los datos no es confiable, ya que presenta valores muy por debajo en contaminación para el conocimiento que si tiene del sistema, recibe aportes directos de agua residual urbana, zonas con gran escorrentía de suelos agrícolas y ganaderos, esto indica que debe existir presencia de contaminantes, posiblemente en cantidades no tan elevadas como las que presenta el modelo.

La recuperación de oxígeno disuelto será alta, los sistemas andinos son muy dinámicos por sus características de pendientes, calados pequeños y elevada turbulencia; esto contribuye a que sea muy elevada la autodepuración, esta 
característica obliga a que exista una gran carga de materia orgánica para la reducción del oxígeno disuelto.

No existe una red de calidad en la microcuenca del río Tabacay que permita tener una información clara de lo que sucede y en qué zona de la microcuenca ocurre. Al tener solo las captaciones como punto de muestreo se tiene una visión global de la problemática pero resulta complicado identificar la zona que está contribuyendo con esta problemática para poder actuar puntualmente, esto hace que se destinen recursos a zonas que probablemente no tienen la misma influencia de contaminación que otras.

El primer paso para lograr modelos de calidad con absoluta fiabilidad es plantear redes de monitoreo que abarquen una amplia zona problema y de esa manera poder ajustar en varios puntos y acercarse a una realidad más global. Esto permitirá una adecuada gestión en lugares afectados por actividades que generen contaminación.

Para la simulación de sólidos suspendidos y bacterias fecales no se depende del oxígeno disuelto por lo que los datos se pueden ajustar a los observados. Se consigue ajustes buenos por lo que para estos parámetros el modelo puede tener la robustez necesaria para predecir concentraciones futuras y servir como herramienta de gestión dentro del Plan de Protección de la microcuenca del río Tabacay.

\section{REFERENCIAS}

[1] Parlamento Europeo y Consejo de la Unión Europea. Directiva Marco, 2000.
[2] The Word Bank (2016) http://http://data.worldbank. org/products/wdi

[3] Paredes, J. Integración de la modelación de la calidad del agua en un sistema de ayuda a la decisión para la gestión del recurso hídrico. Universidad Politécnica de Valencia, 2004.

[4] Torres-Bejarano F., H. Ramírez-León, C. RodríguezCuevas, M. P. Tejera-González y M. C Vásquez Jaraba. Validación de un modelo hidrodinámico y calidad del agua para el Río Magdalena, en el tramo adyacente a Barranquilla, Colombia. Hidrobiológica 25 (1): 7-23. 2015.

[5] M. Martín, P. Marzal, Modelación de la Calidad del Agua. España: Escuela Técnica Superior de Ingenieros de Caminos, Canales y Puertos. Departamento de Ingeniería Hidráulica y Medio Ambiente, 2004.

[6] US Environmental Protection Agency (2005), WASP 7.0. Water Quality Analysis Simulation Program. http: //www.epa.gov/athens/wwptsc/html/wasp.html.

[7] S.C. Chapra, Surface Water Quality Modeling. New York, United States: McGraw-Hill, 1997.

[8] Romero, R. Tratamiento de aguas residuales. Teoría y principios de diseño. Cuarta reimpresión. Editorial Escuala Colombiana de Ingeniería, 2013.

[9] L. Leopold, T. Maddock, The Hydraulic Geometry of Stream Channels and Some Physiographic Implications. United States: Geolical Survey, 1953.

[10] S.H. Bowie, U. Thornton, Environmental Geochemestry and Health. Boston: Reidel Publishing Co, 1985.

Recibido: 01 de Febrero de 2017

Aceptado: 05 de Mayo de 2017

Carlos Matovelle: Máster en Ingeniería Hidráulica y Tratamiento de Agua. Docente de las asignaturas de Modelación Matemática de Sistemas Naturales y a cargo de proyectos de investigación sobre modelación de calidad de agua y determinación de características para sistemas fluviales andinos. 
